\title{
Are premenstrual symptoms associated with health anxiety in nursing graduates?
}

\author{
Yinghui Xu ${ }^{1 *}$, Russell Noyes Jr. ${ }^{2}$, Arthur J. Hartz ${ }^{3}$, Barcey T. Levy ${ }^{1}$, Jeanette M. Daly ${ }^{1}$, \\ Susan R. Johnson ${ }^{4}$ \\ ${ }^{1}$ Department of Family Medicine, Roy J. and Lucille A. Carver College of Medicine, University of Iowa, Iowa City, United States; \\ ${ }^{2}$ Department of Psychiatry, Roy J. and Lucille A. Carver College of Medicine, University of Iowa, Iowa City, United States; \\ ${ }^{3}$ Department of Internal Medicine, University of Utah School of Medicine, Salt Lake City, United States; \\ ${ }^{4}$ Department of Obstetrics \& Gynecology, and Epidemiology, University of Iowa, Iowa City, United States. \\ Email: *yinghui-xu@uiowa.edu
}

Received 25 August 2011; revised 21 September 2011; accepted 29 September 2011.

\begin{abstract}
Objective: This study examined retrospectively the relationship between premenstrual symptoms and health anxiety. Methods: Premenstrual symptoms of nursing school graduates were assessed in 1985 and again in 1991 using the Premenstrual Assessment Form (PAF). A total of 571 women completed the survey in 1991, along with items relating to their physical and mental health. The latter included depression, anxiety, and panic attacks. Health anxiety was also assessed using the Whiteley Index (WI). After women who were amenorrheic for any reason were excluded, a final sample of 410 women aged 25 to 52 years was obtained. Factor analyses yielded 57 items that were useful for calculating a total PAF score. A stepwise multivariate linear regression model was used to find the association of PAF scores with various participant characteristics. Results: Thirtyone of the $410(7.6 \%)$ women had WI scores of $\geq 5$ and were considered to have significant health-related anxiety. The PAF score had statistically significant associations with health anxiety, as well as depression and anxiety. Conclusions: Our results suggest that the premenstrual symptoms often coexist with health anxiety as well as other psychological symptoms. Clinicians should be alert to the fact that PMS may be associated with treatable psychiatric conditions.
\end{abstract}

Keywords: Premenstrual Symptoms; PMDD; Health Anxiety; Hypochondriasis

\section{INTRODUCTION}

Premenstrual syndrome (PMS) is frequently encountered by primary care and specialist physicians. Although as many as $80 \%$ of women experience one or more symptoms [1], clinically significant PMS may occur in $20 \%$ to $50 \%$ of women [2]. Three to $8 \%$ of women are affected by premenstrual dysphoric disorder (PMDD), a more severe form of PMS associated with significant impairment of functioning [1,3-6]. Even though extensive research has been conducted on PMS and PMDD, uncertainty about its etiology and its relationship to biological, psychological and social factors remains.

Psychological factors include stressful life events, personality dimensions, and psychiatric comorbidity. Studies have found high psychiatric comorbidity among women with PMS/PMDD. For instance, $50 \%$ of women with prospectively diagnosed perimenstrual syndromes had concurrent major depression, $35 \%$ had panic disorder, and $20 \%$ had generalized anxiety disorder [4]. The lifetime prevalence of major depression has been found to be $53 \%$ - 58\% in women with prospectively confirmed PMDD [5,7,8], and lifetime anxiety disorders have been observed in $14 \%$ - 16\% of women with PMDD [9].

Health anxiety (hypochondriasis) is another common psychiatric disturbance that is notable for adding to the severity and impairment of other physical and mental disorders. Health anxiety is fear of serious illness arising from bodily sensations. Such anxiety falls on a continuum from mild to severe, and when severe, it is given the designation hypochondriasis [3]. Hypochondriasis is a disorder characterized by heightened attention to bodily sensations that are misinterpreted as signs of serious disease (e.g., this headache means I have a brain tumor). This is a chronic disturbance affecting about $5 \%$ of medical outpatients [10]. Hypochondriasis is associated with increased symptom reporting, impairment in physical and work functioning, and increased health care 
utilization [11]. For instance, recent studies have found that hypochondriasis adds to symptom severity and quality of life in patients with irritable bowel syndrome [12, 13]. In addition, hypochondriasis coexists with other psychiatric disorders. It is found in about $40 \%$ of those with major depression, $10 \%-20 \%$ with panic disorder, $5 \%-10 \%$ with obsessive-compulsive disorder, and generalized anxiety disorder [14].

Despite its importance no research has examined health anxiety in women with PMS/PMDD. Given the relationship between PMS and psychiatric disorders generally, our aim was to examine retrospectively the relationship between premenstrual symptoms and health anxiety.

\section{METHODS}

\subsection{Study Participants}

This study had institutional review board approval, and written informed consent was obtained from all study participants. The original sample consisted of 996 graduates of the University of Iowa College of Nursing, stratified by year of graduation. It included all female graduates from the classes of 1963, 1964, 1965, 1969, 1974, and 1979, as well as sophomore and senior nursing students enrolled in 1984. The first mailing was sent in 1985, and questionnaires were returned by $731(73 \%)$ participants. Those same 731 participants were re-surveyed in 1991, and 571 (78\%) of them responded. Detailed description of two repeat mailings and one telephone contact to non-responders was previously described [15].

Survey participants who were pregnant, postpartum, post-abortion, postmenopausal, who had had a hysterictomy or were without periods for any reason, such as breastfeeding, were excluded. Women who were taking oral contraceptives were not excluded. Final samples consisted of 616 women in 1985 and 410 in 1991.

\subsection{Instruments}

The 1985 questionnaire consisted of 46-items and included information on socio-demographic, menstrual (onset, regularity, duration of cycle, dysmenorrhea, last menstrual period, number of days premenstrual symptoms began before menstrual period, and number of years with PMS), gynecological (diagnosed medical problems, and medications), obstetrical (reproductive events, and oral contraceptive use) characteristics, and the 95-item Premenstrual Assessment Form (PAF) [16]. The questionnaire mailed in 1991 repeated items from 1985 and added 23 items about depression, anxiety, panic attacks, and health anxiety. The latter were elicited with questions such as: "Have you ever suffered from a depression lasting a month or more?", "Have you ever suffered from a period of nervousness or anxiety lasting a month or more?",
"Have you ever suffered from panic attacks?" The possible responses were yes or no. Health anxiety was assessed by the Whiteley Index (WI) of hypochondriasis [17].

The Premenstrual Assessment Form (PAF) is a selfreport questionnaire that elicits information about premenstrual changes in mood, behavior, and physical status. It contains 95 symptoms rated on six-point severity scales from 1 (no change or not applicable) to 6 (extreme change). In answering questions, participants were instructed to consider the condition or change they had experienced during the premenstrual phase of their previous three menstrual cycles. Because there is a considerable variation in the duration of premenstrual phase (range 1 - 14 days), the PAF provides guidelines to help each woman define her premenstrual period and its duration. The PAF can be scored and analyzed in at least three different ways: unipolar dimensional scales, bipolar continua, and typological categories. The numerical scales have been validated by measures of internal consistency, and their reliability is acceptable to good [16].

The Whiteley Index (WI) is a 13-item questionnaire that asks for yes or no responses. In calculating Whiteley Index scores, individual items are scored as 1 (positive) or 0 (negative). All items are scored as positive for an answer of yes except for one, "Is it easy for you to forget about yourself and think about all sorts of other things?" which is reversely scored. The Whiteley Index total score is the sum of all of items with a total possible range of $0-13$. The Whiteley Index has three subscales: bodily preoccupation, disease fear, and disease conviction [17]. However, the three-factor structure has not been confirmed in some studies. As a result, many researchers use the total score of the WI. The internal consistency and test-retest reliability of the WI has been demonstrated to be satisfactory to good, and there is evidence of good concurrent, convergent, and predictive validity [18]. In the present study, a Whiteley Index score of $\geq 5$ was used as the cut-off for health anxiety disorder or hypochondriasis. When the Whiteley Index was used to distinguish between hypochondriacal and non-hypochondriacal patients, as identified by a structured interview using DSM-III-R criteria for hypochondriasis, the sensitivity and specificity were $87 \%$ and $72 \%$ for this cut-off, respectively [19].

\subsection{Statistical Analysis}

PAF data were used for factor analyses to select a subset of inter-correlated items representing PMS. Analyses were originally conducted for PAF data for years 1985 and 1991 separately, and factor structures for those years were similar. The 1985 factor structure was chosen based on the larger sample. The items retained in this factor struc- 
ture were used to calculate PAF summary scores for year 1991.

To identify symptom clusters, an exploratory principal component factor analysis was conducted with varimax rotation. A total of 57 items loading 0.45 or greater were retained. Four factors were readily interpreted that accounted for $17.3 \%, 10.4 \%, 7.7 \%$, and $6.9 \%$ of variance. The first factor, labeled "premenstrual dysphoria", contained 31 items such as, sadness, irritability, and other depressive symptoms. The second factor, labeled "functional impairment", included 12 symptoms, such as avoids activities, stays at home more, and does less housework. Factor three, labeled "motor and cognitive dysfunction", included 8 items such as decreased coordination, has accidents, more forgetful and easily distracted, while factor four labeled, "physical distress", consisted of 6 symptoms, such as bloating, weight gain, abdominal heaviness, and breast pain. Pearson correlations were used to examine the relationships among the four factors. Intercorrelations ranged from the lowest at 0.51 to the highest at 0.75 .

For this study, a total PAF score in year 1991 was calculated by summing all 57 items. Independent variables were also derived from the 1991 questionnaire. The distribution of the PAF total scores approximated a normal curve. Univariate analyses were first used to evaluate the associations between the outcome variable (PAF total) and each independent variable. Student's t-tests or ANOVAs were used to compare scores from different groups. Pearson correlations were computed to examine the associations between pairs of variables. Multivariate regression was used to examine simultaneously the independent relationships between outcome and independent variables. Variables for which univariate analyses indicated p-values of less than 0.10 were selected for inclusion in the multivariate regression model. Stepwise regression was used to select variables using proc REG procedure. A level of 0.05 was specified for a variable to enter or leave the model, and r-squared method was used to select the model. All analyses were performed using SAS (SAS Institute Inc., SAS 9.1.3, Cary, NC).

\section{RESULTS}

In 1991, 410 women between the ages of 25 to 52 years, with a mean age of 39 years $( \pm 7.0 \mathrm{SD})$, were included in the study. The majority were white $(89 \%)$ and married or living as married (77\%). Most of them (93\%) had graduated from college and $87 \%$ were employed.

The mean duration of premenstrual symptoms was 6.0 days $( \pm 3.1 \mathrm{SD})$ with a range from 1 to 14 days before the menses. The mean PAF score was 94.4 ( \pm 38.9 SD) with a range from 57 to 258 . Younger women had signifi- cantly higher mean PAF scores than older women (Table 1). Women who had medical problems and were currently under a doctor's care as well as those taking medications had significantly higher PAF scores than did those without medical problems. Mean PAF scores were significantly higher among women with endometriosis, dysmenorrhea, and migraine compared with participants without these problems. No significant differences were

Table 1. PAF mean scores ${ }^{*}$ according to patient characteristics.

\begin{tabular}{|c|c|c|c|}
\hline & N (\%) & $\begin{array}{l}\text { PAF Mean } \\
\text { Scores }\end{array}$ & p-values \\
\hline Age (years) & & & 0.023 \\
\hline $25-29$ & $58(14.8 \%)$ & 100.0 & \\
\hline $30-39$ & $173(44.0 \%)$ & 98.0 & \\
\hline $40-52$ & $162(41.2 \%)$ & 87.8 & \\
\hline Marital Status & & & 0.873 \\
\hline With a partner & $222(76.6 \%)$ & 96.8 & \\
\hline Without a partner & $68(23.4 \%)$ & 97.7 & \\
\hline Annual Household Income & & & 0.168 \\
\hline$<\$ 34,999$ & $123(38.1 \%)$ & 99.4 & \\
\hline$\$ 35,999-\$ 99,999$ & $177(54.8 \%)$ & 90.0 & \\
\hline$>\$ 100,000$ & $23(7.1 \%)$ & 90.4 & \\
\hline Education & & & 0.104 \\
\hline$<16$ years & $27(7.3 \%)$ & 105.0 & \\
\hline$\geq 16$ years & $341(92.7 \%)$ & 92.6 & \\
\hline Smoking & & & 0.414 \\
\hline Current & $37(9.0 \%)$ & 99.4 & \\
\hline Non-smoker & $373(91.0 \%)$ & 93.9 & \\
\hline Current Medical Problems & & & 0.023 \\
\hline Yes & $103(25.1 \%)$ & 103.0 & \\
\hline No & $307(74.9 \%)$ & 91.6 & \\
\hline Number of Current Medications & & & $<0.001$ \\
\hline None & $324(79.0 \%)$ & 88.9 & \\
\hline One or more & $69(16.8 \%)$ & 111.6 & \\
\hline Regular Menstruation & & & 0.226 \\
\hline Yes & $312(76.8 \%)$ & 92.7 & \\
\hline No & $94(23.2 \%)$ & 98.3 & \\
\hline Tubal Ligation & & & 0.597 \\
\hline Yes & $88(21.5 \%)$ & 92.5 & \\
\hline No & $322(78.5 \%)$ & 95.0 & \\
\hline Taking Birth Control Pill & & & 0.772 \\
\hline Yes & $50(12.2 \%)$ & 95.9 & \\
\hline No & $360(87.8 \%)$ & 94.2 & \\
\hline Ever Pregnant & & & 0.025 \\
\hline Yes & $316(77.1 \%)$ & 95.6 & \\
\hline No & $94(22.9 \%)$ & 113.4 & \\
\hline Endometriosis & & & 0.049 \\
\hline Yes & $19(5.0)$ & 119.1 & \\
\hline No & $361(95.0)$ & 93.3 & \\
\hline Dysmenorrhea & & & $<0.001$ \\
\hline Yes & $94(23.0)$ & 110.1 & \\
\hline No & $314(77.0)$ & 89.5 & \\
\hline Migraine & & & 0.014 \\
\hline Yes & $45(11.8)$ & 110.9 & \\
\hline No & $335(88.2)$ & 92.4 & \\
\hline
\end{tabular}

*Scores ranged from 57 to 258 with higher scores indicating more PMS symptoms. 
observed in PAF scores according to marital status, income, education, smoking status, regularity of menses, history of tubal ligation, or taking birth control pills.

PAF scores according to the presence or absence of individual psychiatric conditions were examined (Table 2). Mean PAF scores were significantly higher among women with histories of depression, anxiety, and panic attacks, as well as with family histories of anxiety or depression.

The mean of Whiteley Index total score for the sample was 1.7 with a range of $0-12$. Thirty-one of the 410 (7.6\%) women had a Whiteley Index score of $\geq 5$ and were considered to have health anxiety disorder or hypochondriasis [19]. The mean PAF score for this group was significantly higher than that from those without health anxiety $(119 \pm 55.3(\mathrm{n}=31)$ vs. $92.4 \pm 36.6(\mathrm{n}=379)$; t-test, $\mathrm{p}=0.01)$. Positive responses on some Whiteley Index items were associated with higher mean PAF scores (Table 3). For example, women who answered the following questions positively had significantly higher PAF scores: Are you bothered by many different symptoms? Is it hard for you to believe the doctor when he tells you that you have nothing to worry about? Do you worry a lot about your health? Do you worry about the possibility that you have a serious illness? Are you often aware of various things happening in your body? PAF scores were consistently higher among those answering questions positively compared with those answering negatively, with the exception of the question: Are you afraid of illness? Significant correlations were observed between mean PAF scores and bodily preoccupation $(\mathrm{r}=$ $0.2119, \mathrm{p}<0.0001)$, disease conviction $(\mathrm{r}=0.1861, \mathrm{p}<$ $0.001)$, but not disease phobia $(\mathrm{r}=0.0858, \mathrm{p}=0.0829)$ subscales of the Whiteley Index.

Variables that showed significant association $(\mathrm{p}<0.10)$ with PAF scores in the univariate analysis were examined further using multivariate regression (Table 4). Variables significantly and independently associated

Table 2. PAF mean scores according to the presence or absence of psychiatric conditions.

\begin{tabular}{lcl}
\hline & \multicolumn{2}{c}{ Mean $(\mathrm{n})$} \\
\hline Variables & Presence & Absence \\
\hline
\end{tabular}

Psychiatric Conditions

History of Depression $\quad 108.3(\mathrm{n}=93) \quad 90.3(\mathrm{n}=317) \quad 0.001$

History of Anxiety $\quad 116.1(\mathrm{n}=37) \quad 92.3(\mathrm{n}=373) \quad 0.016$

History of Panic Attacks $\quad 115.3(\mathrm{n}=47) \quad 91.7(\mathrm{n}=363) \quad 0.002$

Family History of Anxiety or $105.1(\mathrm{n}=101) 90.2(\mathrm{n}=293) \quad 0.004$

History of Post-partum $\quad 98.5(\mathrm{n}=30) \quad 94.1(\mathrm{n}=380) \quad 0.548$

Table 3. PAF mean scores according to the responses to whiteley index questions.

\begin{tabular}{|c|c|c|c|}
\hline \multirow[b]{2}{*}{ Questions } & \multicolumn{2}{|c|}{ Mean (n) } & \multirow{2}{*}{ p-values } \\
\hline & Positive & Negative & \\
\hline Are you bothered by many different symptoms? & $138.3(n=24)$ & $92.0(\mathrm{n}=381)$ & $<0.001$ \\
\hline Do you get the feeling that people are not taking your illness seriously enough? & $134.9(\mathrm{n}=12)$ & $93.3(n=395)$ & 0.065 \\
\hline Is it hard for you to believe doctor when he tells you nothing to worry about? & $121.2(\mathrm{n}=22)$ & $93.1(n=384)$ & 0.041 \\
\hline Do you think something serious wrong with your body? & $117.3(\mathrm{n}=21)$ & $93.2(\mathrm{n}=387)$ & 0.058 \\
\hline Do you worry about the possibility that you got a serious illness? & $111.6(n=27)$ & $93.3(\mathrm{n}=381)$ & 0.018 \\
\hline Is it easy for you to forget about yourself? & $109.2(\mathrm{n}=34)$ & $93.0(n=374)$ & 0.078 \\
\hline If a disease is brought to your attention, do you worry about getting it yourself? & $107.7(\mathrm{n}=26)$ & $93.5(n=383)$ & 0.072 \\
\hline If you feel ill and someone tells you that you are looking better, you become annoyed. & $103.6(\mathrm{n}=38)$ & $93.5(\mathrm{n}=372)$ & 0.128 \\
\hline Do you worry about your health more than other people? & $101.9(n=20)$ & $94.0(n=390)$ & 0.530 \\
\hline Are you bothered by many pains? & $98.5(\mathrm{n}=84)$ & $93.4(\mathrm{n}=326)$ & 0.282 \\
\hline Are you afraid of illness? & $94.4(\mathrm{n}=114)$ & $94.8(\mathrm{n}=292)$ & 0.929 \\
\hline
\end{tabular}

Table 4. Final regression model associated with PAF score.

\begin{tabular}{lccc}
\hline Variables & Coefficients & t-values & p-values \\
\hline History of panic attacks (yes vs. no) & 19.8 & 3.44 & 0.001 \\
History of depression (yes vs. no) & 17.0 & 3.78 & $<0.001$ \\
Whiteley Index total score & 2.9 & 2.82 & 0.005 \\
Age (Years) & -0.7 & -2.57 & 0.011 \\
\hline
\end{tabular}

with higher PAF scores were history of depression, history of panic attacks, total Whiteley Index score and age. The coefficients represented the expected change in PAF score associated with a one-unit change in each variable, given the other variables in the model. For example, the PAF score was 19.8 points higher in women who had a history of panic attacks than it was in those without a 
history of panic attacks. The PAF score was 0.7 points lower for each one year increase in age. The r-squared for the model was 0.12 , thus $12 \%$ of the variance of PAF score was explained by the variables in this model.

\section{DISCUSSION}

In the current study, we found health anxiety, as measured by the Whiteley Index, associated with the premenstrual symptoms. In addition, a history of depression, anxiety and younger age were also associated with such symptoms. To our knowledge, this is the first demonstration of such a link. Given the relationship of health anxiety with other symptomatic and functional disturbances, this finding may not seem surprising. It does not, of course, establish cause. Such anxiety may serve to accentuate the experience and reporting of premenstrual symptoms, or premenstrual symptoms may lead to anxiety about one's health, especially if they are not as is too often the case correctly identified and treated. Either way, the presence of health anxiety was associated with distress and impairment associated with premenstrual symptoms.

Earlier studies have found that health anxiety tends to be high among medical specialty populations in which patients with functional and psychiatric disturbances are frequent. For instance, one survey found hypochondriasis (health anxiety) in 13 percent of otolaryngology clinic patients [20]. Also, hypochondriacal symptoms are higher in patients with functional and psychiatric disturbances than in those with organic illnesses. For example, one study found significantly higher hypochondriasis scores in patients with irritable bowel syndrome than in those with organic gastrointestinal disease [21]. Health anxiety and hypochondriasis tend to be associated with increased symptom reporting and functional impairment in clinical populations. One study found hypochondriasis in $50 \%$ of patients in a pain clinic [22], and another found high health anxiety predictive of abdominal pain a year later [23]. Still another found hypochondriasis associated with chronic pain and pain intensity [24]. Of course, these associations do not establish cause; painful symptoms may lead to anxiety about health.

Our finding of significant association between premenstrual symptoms and both depression and anxiety are consistent with earlier studies [4,7,9,25]. However, as in previous retrospective studies [26,27], we were not able to determine the temporal relationship between these disorders and PMS. Consequently, it is difficult to know which may have contributed to the other. Indeed, a definitive diagnosis of PMDD requires prospective charting of mood and somatic symptoms in two consecutive menstrual cycles, and it can be challenging to distinguish the symptoms of PMDD from those of other psychiatric disorders.
We found that pain syndromes, such as endometriosis, dysmenorrhea, and migraine, were also significantly associated with premenstrual symptoms in univariate analysis. The diagnoses of these conditions were based on self-report, and thus mis-classification may have occurred. Also, we did not use a standardized instrument to collect these diagnoses, so this data can not be compared directly to other studies. Endometriosis is an estrogen-dependent disease, the symptoms of which include chronic pelvic pain, dysmenorrhea and infertility. Endometriosis and PMS are both hormonally influenced conditions with a wide spectrum severity related to the menstrual cycle. Dysmenorrhea has also been linked to PMS [28,29] as has migraine in a few studies. For instance, Hutchinson and colleagues [30] reported that women suffering from migraine also frequently observe headache during PMS. Most recently, Martin et al. [31] found that premenstrual symptoms were more severe when patients were experiencing migraine. The association between these pain syndromes and PMS is consistent with the hypothesis that these syndromes maybe linked to fluctuations in the levels of estrogen and progesterone [32].

However, it is important to note that endometriosis, dysmenorrhea, and migraine did not remain in the final regression model after controlling for psychiatric disorders. Psychiatric disorders are themselves associated with increased reporting of physical symptoms. For example, patients with major depression experience more pain symptoms than those without depression [33-35]. Conversely, patients with chronic pain frequently develop depressive disorders, and successful treatment of depression is often associated with improvement in the pain [36,37]. Thus, pain syndromes and psychiatric disorders interact and this may explain the failure of such syndromes to appear in the final model.

Although the reliability and validity of the 95-item PAF have been tested, the instrument has been criticized for the overlap in its categorical and numerical scales [38]. We, therefore, sought to find items that most accurately reflected the premenstrual symptoms in this large and well-educated group of women, and identified four factors: premenstrual dysphoria, functional impairment, motor and cognitive dysfunction, and physical distress. Premenstrual dysphoria was the dominant factor, which accounted for 17.3 percent of the variance. Symptoms that loaded most strongly on this factor included sadness, irritability, depression, inability to cope and loneliness. The factors identified in our analysis are comparable to those found in several previous studies [39-41]. Also, the symptoms of premenstrual dysphoria as well as physical distress extracted in our study were also very similar to 
those found in these studies. Premenstrual dysphoria has consistently been identified as the first factor in these studies; it appears to be the central or core feature of PMDD.

This study had several strengths as well as limitations. The strengths included a large group of well-educated women aware of health issues by virtue of their study of nursing. In addition, the study was conducted before selective serotonin re-uptake inhibitors were in widespread use, thereby eliminating a confounding factor that would complicate such a study if done today. This study also has several limitations. First, participants were largely Caucasian, college graduates, and members of the middle class. Consequently, they may not have been representative of the general population. Second, this was a retrospective study, in which the symptoms were not confirmed by daily symptom ratings that are the current gold standard for diagnosis of PMS/PMDD. Therefore, the diagnosis of PMDD based on DSM-IV criteria was not possible. In a retrospective study of PMS, women were more likely to exaggerate past symptoms and negative moods [42]. Our participants may also have over-reported symptoms. Third, we did not exclude women with any psychiatric disorders, and these disorders may have inflated premenstrual symptoms. Lastly, the most important risk factors, such as depression, anxiety, panic attacks, and health anxiety were only collected in 1991. Therefore, we could not examine covariates of premenstrual symptoms or risk factors over time.

Health anxiety (hypochondriasis) is important because it is prevalent, is associated with significant impairment and is treatable. Effective management and treatment, including psychological therapies and medication, are relatively recent developments. Clinicians should be aware of the association of health anxiety with PMS/ PMDD. Such anxiety as well as other psychiatric disorders are often masked by the presentation of premenstrual and other physical symptoms. However, if inquiry is directed toward the meaning of symptoms, health worry and fear of serious illness may be elicited. Health anxiety may respond to education regarding premenstrual and psychiatric symptoms, but definitive treatments include cognitive behavioral therapy and serotonin re-uptake inhibitors. The latter are used in the treatment of PMS/PMDD but are often used intermittently. For health anxiety and other psychiatric disorders continuous administration is necessary.

Prospective studies will be needed to clarify the relationship between health anxiety, other psychiatric disorders, and PMS and PMDD.

\section{DECLARATION OF INTEREST}

The authors declare that they have no competing interests.

\section{REFERENCES}

[1] Steiner, M., Macdougall, M. and Brown, E. (2003) The premenstrual symptoms screening tool (PSST) for clinicians. Arch Women's Mental Health, 6, 203-209. doi:10.1007/s00737-003-0018-4

[2] Gehlert, S., Chang, C.H. and Hartlage, S. (1999) Symptom patterns of premenstrual dysphoric disorder as defined in the diagnostic and statistical manual of mental disorders-IV. Journal of Women's Health, 8, 75-85. doi:10.1089/jwh.1999.8.75

[3] American Psychiatric Association (APA). (2000) Diagnostic and statistical manual of mental disorders. 4th Edition, American Psychiatric Association, Washington DC.

[4] Angst, J., Sellaro, R., Merikangas, K.R. and Endicott, J. (2001) The epidemiology of perimenstrual psychological symptoms. Acta Psychiatric Scand, 104, 110-116. doi:10.1034/j.1600-0447.2001.00412.x

[5] Cohen, L.S., Soares, C.N., Otto, M.W., Sweeney, B.H., Liberman, R.F. and Harlow, B.L. (2002) Prevalence and predictors of premenstrual dysphoric disorder (PMDD) in older premenopausal women, the Harvard study of moods and cycles. Journal of Affect Disorders, 70, 125132. doi:10.1016/S0165-0327(01)00458-X

[6] Wittchen, H.U., Becker, E., Lieb, R. and Krause, P. (2002) Prevalence, incidence and stability of premenstrual dysphoric disorder in the community. Psychological Medicine, 32, 119-132.

doi:10.1017/S0033291701004925

[7] Critchlow, D.G., Bond, A.J. and Wingrove, J. (2001) Mood disorder history and personality assessment in premenstrual dysphoric disorder. Journal of Clinical Psychiatry, 62, 688-693. doi:10.4088/JCP.v62n0905

[8] Soares, C.N., Cohen, L.S., Otto, M.W. and Harlow, B.L. (2001) Characteristics of women with premenstrual dysphoric disorder (PMDD) who did or did not report history of depression: A preliminary report from the harvard study of moods and cycles. Journal of Women's Health Gender-Based Medicine, 10, 873-878. doi:10.1089/152460901753285778

[9] Yonkers, K.A. (1997) Anxiety symptoms and anxiety disorders: How are they related to premenstrual disorders? The Journal of Clinical Psychiatry, 58, S62- S67.

[10] Noyes, R. (2009) Hypochondriasis. New Oxford textbook of psychiatry. 2nd Edition, Oxford University Press, New York.

[11] Taylor, S. and Asmundson, G.J.G. (2004) Treating health anxiety: A cognitive-behavioral approach. Guilford, New York.

[12] Gros, D.F., Antony, M.M., McCabe, R.E. and Swinson, R.P. (2009) Frequency and severity of the symptoms of irritable bowel syndrome across the anxiety disorders and depression. Journal Anxiety Disorders, 23, 290-296. doi:10.1016/j.janxdis.2008.08.004

[13] Rey, E., Garcia-Alonso, M.O., Moreno-Ortega, M., Alvarez-Sanchez, A. and Diaz-Rubio, M. (2008) Determinants of quality of life in irritable bowel syndrome. Journal of Clinical Gastroenterology, 42, 1003-1039. doi:10.1097/MCG.0b013e31815af9f1

[14] Barsky, A.J. (2001) Clinical practice. The patient with hypochondriasis. New England Journal of Medicine, 345, 
1395-1399. doi:10.1056/NEJMcp002896

[15] Johnson, S.R., McChesney, C. and Bean, J.A. (1988) Epidemiology of premenstrual symptoms in a nonclinical sample. I. prevalence, natural history and help-seeking behavior. Journal of Reproductive Medicine, 33, 340346.

[16] Halbreich, U., Endicott, J., Schacht, S. and Nee, J. (1982) The diversity of premenstrual changes as reflected in the premenstrual assessment form. Acta Psychiatric Scand, 65, 46-65. doi:10.1111/j.1600-0447.1982.tb00820.x

[17] Pilowsky, I. and Spence, N.D. (1976) Manual for the illness behavioural questionnaire (IBQ). 2nd Edition, Department of Psychiatry, University of Adelaide, South Australia.

[18] Speckens, A.E. (2001) Assessment of hypochondriasis. Modern perspectives on an ancient malady. Oxford University Press, New York, 2001.

[19] Speckens, A.E., Van Hemert, A.M., Spinhoven, P. and Bolk, J.H. (1996) The diagnostic and prognostic significance of the whitely index, the illness attitude scales and the somatosensory amplification scale. Psychological Medicine, 26, 1085-1090. doi:10.1017/S0033291700035418

[20] Schmidt, A.J., van Roosmalen, R., van der Beek, J.M. and Lousberg, R. (1993) Hypochondriasis in ENT practice. Clinical Otolaryngology, 18, 508-511. doi:10.1111/j.1365-2273.1993.tb00625.x

[21] Gomborone, J., Dewsnap, P., Libby, G. and Farthing, M. (1995) Abnormal illness attitudes in patients with irritable bowel syndrome. Journal of Psychosomatic Research, 39, 227-230. doi:10.1016/0022-3999(94)00126-P

[22] Wong, W.S., Chen, P.P., Yap, J., Mak, K.H., Tam, B.K. and Fielding, R. (2011) Chronic pain and psychiatric morbidity: A comparison between patients attending specialist orthopedics clinic and multidisciplinary pain clinic. Pain Medicine, 12, 246-259. doi:10.1111/j.1526-4637.2010.01044.x

[23] Halder, S.L., McBeth, J., Silman, A.J., Thompson, D.G. and Macfarlane, G.J. (2002) Psychosocial risk factors for the onset of abdominal pain. Results from a large prospective population-based study. International Journal of Epidemiology, 31, 1219-1225, Discussion 1225-1226.

[24] Fishbain, D.A., Lewis, J.E., Gao, J., Cole, Steele, B. and Rosomoff, R. (2009) Is chronic pain associated with somatization/hypo-chondriasis? An evidence-based structured review. Pain Practice, 9, 449-467. doi:10.1111/j.1533-2500.2009.00309.x

[25] Yonkers, K.A. (1997) The association between premenstrual dysphoric disorder and other mood disorders. Journal of Clinical Psychiatry, 58, 19-25.

[26] Adewuya, A.O., Loto, O.M. and Adewumi, T.A. (2008) Premenstrual dysphoric disorder amongst nigerian university students: Prevalence, comorbid conditions, and correlates. Arch Women's Mental Health, 11, 13-18. doi:10.1007/s00737-008-0213-4

[27] Breaux, C., Hartlage, S. and Gehlert, S. (2000) Relationships of premenstrual dysphoric disorder to major depression and anxiety disorders: A re-examination. Journal of Psychosomatic Obstetric Gynecology, 21, 17-24. doi:10.3109/01674820009075604

[28] Vichnin, M., Freeman, E.W., Lin, H., Hillman, J. and Bui, S. (2006) Premenstrual syndrome (PMS) in adolescents:
Severity and impairment. Journal of Pediatric and Adolescent Gynecology, 19, 397-402.

doi:10.1016/j.jpag.2006.06.015

[29] Nisar, N., Zehra, N., Haider, G., Munir, A.A. and Sohoo, N.A. (2008) Frequency, intensity and impact of premenstrual syndrome in medical students. Journal of College of Physicians and Surgeons Pakistan, 18, 481-484.

[30] Hutchinson, S.L. and Silberstein, S.D. (2008) Menstrual migraine: Case studies of women with estrogen-related headaches. Headache, 48, S131-S141. doi:10.1111/j.1526-4610.2008.01311.x

[31] Martin, V.T., Wernke, S., Mandell, K., Ramadan, N., Kao, L., Bean, J., Liu, J., Zoma, W. and Rebar, R. (2006) Symptoms of premenstrual syndrome and their association with migraine headache. Headache, 46, 125-137. doi:10.1111/j.1526-4610.2006.00306.x

[32] Farage, M.A., Osborn, T.W. and MacLean, A.B. (2008) Cognitive, sensory, and emotional changes associated with the menstrual cycle: A review. Arch Gynecology and $\mathrm{Ob}$ stetrics, 27, 299-307. doi:10.1007/s00404-008-0708-2

[33] Arnow, B.A., Hunkeler, E.M., Blasey, C.M., Lee, J., Constantino, M.J., Fireman, B., Kraemer, H.C., Dea, R., Robinson, R. and Hayward, C. (2006) Comorbid depression, chronic pain, and disability in primary care. Psychosomatic Medicine, 68, 262-268. doi:10.1097/01.psy.0000204851.15499.fc

[34] Bair, M.J., Robinson, R.L., Katon, W. and Kroenke, K. (2003) Depression and pain comorbidity: A literature review. Arch International Medicine, 163, 2433-2445. doi:10.1001/archinte.163.20.2433

[35] McWilliams, L.A., Cox, B.J. and Enns, M.W. (2003) Mood and anxiety disorders associated with chronic pain: An examination in a nationally representative sample. Pain, 106, 127-133. doi:10.1016/S0304-3959(03)00301-4

[36] Gureje, O., Simon, G.E. and Von Korff, M. (2001) A cross-national study of the course of persistent pain in primary care. Pain, 92, 195-200. doi:10.1016/S0304-3959(00)00483-8

[37] Gureje, O., Von Korff, M., Simon, G.E. and Gater, R. (1998) Persistent pain and well-being: A world health organization study in primary care. The Journal of the American Medical Association, 280, 147-151. doi:10.1001/jama.280.2.147

[38] Cumming, D.C., Cumming, C.E., Krausher, R.J. and Fox, E.E. (1991) Towards a definition of PMS II: A factor analytic evaluation of premenstrual change in women with symptomatic premenstrual change. Journal of Psychosomatic Research, 35, 713-720. doi:10.1016/0022-3999(91)90122-5

[39] Feuerstein, M. (2002) Measurement properties of the calendar of premenstrual experience in patients with premenstrual syndrome. Journal of Reproductive Medicine, 4, 279-289.

[40] Freeman, E.W., DeRubeis, R.J. and Rickels, K. (1996) Reliability and validity of a daily diary for premenstrual syndrome. Psychiatry Research, 65, 97-106. doi:10.1016/S0165-1781(96)02929-0

[41] Teng, C.T., Filho, A.H., Artes, R., Gorenstein, C., Andrade, L.H. and Wang, Y.P. (2005) Premenstrual dysphoric symptoms amongst brazilian college students: Factor structure and methodological appraisal. European Arch Psychiatry 
Clinical Neuroscience, 255, 51-56.

doi:10.1007/s00406-004-0535-9

[42] Marvan, M.L. and Cortes-Iniestra, S. (2001) Women's beliefs about the prevalence of premenstrual syndrome and biases in recall of premenstrual changes. Health Psychology, 20, 276-280.

doi:10.1037/0278-6133.20.4.276 